\title{
Caffeine inhibits adipogenesis through modulation of mitotic clonal expansion and the AKT/GSK3 pathway in 3T3-L1 adipocytes
}

\author{
Hyo Jung Kim ${ }^{1}$, Bo Kyung Yoon ${ }^{2}$, Hyounkyoung Park ${ }^{1}$, Jo Woon Seok ${ }^{1,3}$, Hyeonjin Choi ${ }^{1}$, Jung Hwan Yu ${ }^{1,3}$, Yoonjeong Choi ${ }^{1,3}$, \\ Su Jin Song ${ }^{1,3}$, Ara Kim ${ }^{1,3}$ E Jae-woo Kim ${ }^{1,3,4, *}$ \\ ${ }^{1}$ Department of Biochemistry and Molecular Biology, Integrated Genomic Research Center for Metabolic Regulation, Institute of Genetic \\ Science, Yonsei University College of Medicine, ${ }^{2}$ Yonsei University College of Medicine, ${ }^{3}$ Brain Korea 21 PLUS Project for Medical Science, \\ Yonsei University, ${ }^{4}$ Severance Biomedical Science Institute, Yonsei University College of Medicine, Seoul 03722, Korea
}

\begin{abstract}
Caffeine has been proposed to have several beneficial effects on obesity and its related metabolic diseases; however, how caffeine affects adipocyte differentiation has not been elucidated. In this study, we demonstrated that caffeine suppressed 3T3-L1 adipocyte differentiation and inhibited the expression of CCAAT/enhancer binding protein (C/EBP) $\alpha$ and peroxisome proliferator-activated receptor (PPAR) $\gamma$, two main adipogenic transcription factors. Anti-adipogenic markers, such as preadipocyte secreted factor (Pref)-1 and Krüppel-like factor 2, remained to be expressed in the presence of caffeine. Furthermore, 3T3-L1 cells failed to undergo typical mitotic clonal expansion in the presence of caffeine. Investigation of hormonal signaling revealed that caffeine inhibited the activation of AKT and glycogen synthase kinase (GSK) 3 in a dose-dependent manner, but not extracellular signal-regulated kinase (ERK). Our data show that caffeine is an anti-adipogenic bioactive compound involved in the modulation of mitotic clonal expansion during adipocyte differentiation through the AKT/GSK3 pathway. [BMB Reports 2016; 49(2): 111-115]
\end{abstract}

\section{INTRODUCTION}

Obesity is a strong risk factor for metabolic disorders including hypertension, dyslipidemia, cardiovascular disease, and type 2 diabetes, and has become a worldwide public health problem $(1,2)$. Obesity is associated with an excessive growth and expansion of adipose tissue mass through increases in both the

*Corresponding author. Tel: +82-2-2228-0837; Fax: +82-2-312-5041; E-mail: japol13@yuhs.ac

http://dx.doi.org/10.5483/BMBRep.2016.49.2.128

Received 26 June 2015, Revised 27 July 2015 , Accepted 9 September 2015

Keywords: Adipogenesis, AKT, Caffeine, Glycogen synthase kinase, Mitotic clonal expansion number and size of fat cells (3). Therefore, many studies have been performed to identify critical processes for either reducing lipid accumulation or inhibiting adipocyte formation to limit obesity and its metabolic consequences.

3T3-L1 is a well-established preadipocyte cell line for the study of adipogenesis (4). Upon a hormonal stimulation with 1-methyl 3-isobutylxanthine, dexamethasone, and insulin, growth-arrested 3T3-L1 preadipocytes synchronously reenter the cell cycle, known as mitotic clonal expansion, and then express adipocyte-specific genes for terminal differentiation $(5,6)$. Adipogenic differentiation is regulated by a cascade of several transcription factors including CCAAT/enhancer-binding protein (C/EBP) family members and peroxisome proliferator-activated receptor (PPAR) $\gamma(7,8)$. During mitotic clonal expansion, both $\mathrm{C} / \mathrm{EBP} \beta$ and $\mathrm{C} / \mathrm{EBP} \delta$ are temporally expressed and regulate cell proliferation $(9,10)$. This process eventually leads to the expression of $\mathrm{C} / \mathrm{EBP} \alpha$ and PPAR $\gamma$, two pleiotropic transcriptional activators that coordinately induce terminal differentiation to a mature adipocyte phenotype by regulating adipocyte-specific genes. The transition from mitotic clonal expansion to terminal differentiation involves several mechanisms, including the phosphorylation $(11,12)$, dimerization (13), and reactive oxygen species (ROS)-mediated activation (5) of C/EBP 3 . Also, factors regulating the adipogenic process, either positively or negatively, have been identified such as preadipocyte secreted factor (Pref)-1 (14) and Krüppel-like factors (15-17).

Caffeine (1,3,7-trimethylxanthine) is a plant alkaloid found in coffee, chocolate, and tea, widely consumed by humans worldwide. Several studies have shown that caffeine reduces body weight and adipose tissue weight in animal models (18). Moreover, caffeine has a variety of effects on metabolism; it increases energy expenditure by inhibiting the phosphodiesterase-induced degradation of intracellular cAMP, results in reduced food intake, alters intracellular calcium levels, increases cAMP-dependent protein kinase (PKA), and inhibits phosphatidylinositol-3-kinase (PI3K)/AKT activity (19). It has recently been demonstrated that caffeine suppresses intracellular lipid accumulation $(20,21)$, inhibits insulin-stimulated glucose uptake,

ISSN: 1976-670X (electronic edition)

Copyright (c) 2016 by the The Korean Society for Biochemistry and Molecular Biology

(c) This is an open-access article distributed under the terms of the Creative Commons Attribution Non-Commercial License (http://creativecommons.org/licenses/by-nc/4.0) which permits unrestricted non-commercial use, distribution, and reproduction in any medium, provided the original work is properly cited. 
and increases lipolysis in adipose cells (22). These results indicate that caffeine plays a role in reducing lipid accumulation; however, how caffeine affects adipogenesis has not been clearly elucidated.

In this study, we show that caffeine inhibits the expression of C/EBP $\beta, C / E B P \alpha$, and PPAR $\gamma$ during 3T3-L1 preadipocyte differentiation. Caffeine exerted its inhibitory effects on adipocyte differentiation by regulating the expression of G1-S phase-specific cell cycle markers, thereby blocking mitotic clonal expansion. Our results demonstrate that caffeine has an anti-adipogenic effect on 3T3-L1 preadipocytes that involves the AKT/ glycogen synthase kinase (GSK) $3 \beta$ pathway.

\section{RESULTS}

\section{Caffeine inhibits 3T3-L1 differentiation in a dose-dependent manner}

To elucidate the effects of caffeine and its underlying molecular mechanisms, we first tested the effect of caffeine on adipocyte differentiation in 3T3-L1 cells. 3T3-L1 cells were incubated in adipogenic differentiation medium in the presence of increasing concentrations of caffeine for 8 days, resulting in a dose-dependent inhibition of adipocyte differentiation; at 5 $\mathrm{mM}$ of caffeine, differentiation was almost completely suppressed (Fig. 1A). To quantify the magnitude of adipocyte dif-

A

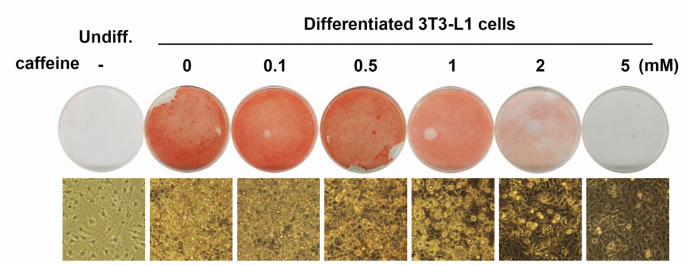

B

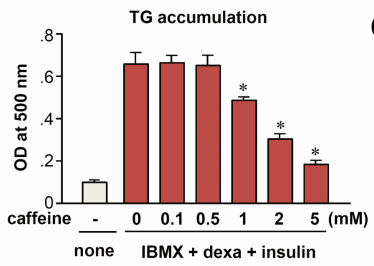

C

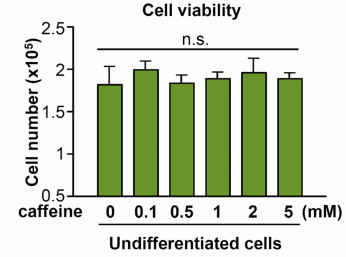

Fig. 1. Caffeine inhibits adipocyte differentiation in a dose-dependent manner. (A) Two-day post-confluent 3T3-L1 preadipocytes incubated with the hormonal inducers (IBMX, dexamethasone, and insulin) in the absence or presence of caffeine. After 2 days, all groups were fed with FBS, DMEM, and insulin for another 2 days and then with FBS and DMEM. After 8 days of differentiation, lipid accumulation was detected by oil-red-O staining. Representative photomicrographs $(\times 400)$ are shown for each treatment group. (B) Quantitative analysis of adipocyte differentiation, assessed by spectrophotometric measurement of oil-red-O-stained adipocytes. ${ }^{*} \mathrm{P}<0.01$ vs. $0 \mathrm{mM}$ caffeine group. (C) Cell count was performed in 3T3-L1 preadipocytes in which cells were incubated with the indicated concentration of caffeine for $48 \mathrm{~h}$ in growth medium in the absence of MDI. n.s., not significant. ferentiation, oil-red-O-stained cells were extracted in isopropyl alcohol and measured at $500 \mathrm{~nm}$ using a spectrophotometer. Consistent with the results of microscopic examination, the amount of oil-red-O stain eluted from cells was significantly decreased in samples treated with $>1 \mathrm{mM}$ caffeine (Fig. 1B). As shown in Fig. 1C, caffeine did not significantly affect cell viability at concentrations up to $5 \mathrm{mM}$. Thus, caffeine was used in a range of non-cytotoxic concentrations (0.1 to $5 \mathrm{mM})$ in subsequent experiments.

Adipogenesis, the process by which adipocyte precursors develop into mature adipocytes, is regulated by a group of specific transcription factors such as PPAR $\gamma$ and C/EBP $\alpha$ (4). To confirm whether diminished accumulation of triglyceride after caffeine exposure was accompanied by a change in protein expression for adipocyte-specific genes, whole cell lysates were obtained and subjected to western blot analysis. Our results showed that the protein expression of $\mathrm{C} / \mathrm{EBP} \beta, \mathrm{C} / \mathrm{EBP} \alpha$, and PPAR $\gamma$ were decreased after caffeine treatment in a dose-dependent manner (Fig. 2A). The mRNA expression of adipocytespecific genes, such as aP2/422 and FAS, was also diminished. In contrast, inhibitory factors of adipogenesis remained high (KLF2 and Pref-1) with comparable treatment (Fig. 2B). These results suggest that caffeine inhibits adipogenic differentiation accompanied by the suppression of C/EBP $\alpha$ and PPAR $\gamma$ expression. Interestingly, C/EBP $\beta$ expression was not affected by $1 \mathrm{mM}$ caffeine treatment, at which concentration the expression of C/EBP $\alpha, \mathrm{PPAR} \gamma, \mathrm{aP} 2 / 422, \mathrm{KLF} 2$, and Pref- 1 was affected, suggesting that $\mathrm{C} / \mathrm{EBP} \beta$ expression is not a primary target for caffeine.

\section{Caffeine inhibits the mitotic clonal expansion process in 3T3-L1 preadipocytes}

To examine the role of caffeine in adipogenesis, we assessed

A

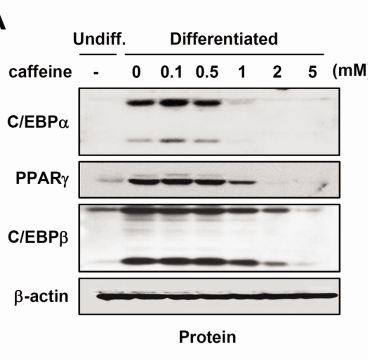

B

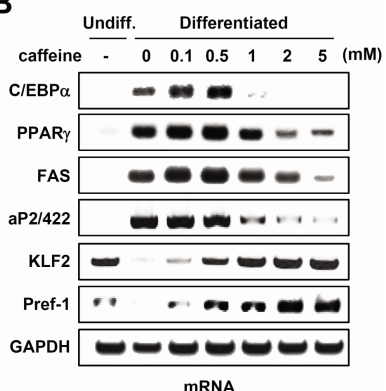

Fig. 2. The effect of caffeine on the expression of adipogenic marker genes. Two-day post-confluent 3T3-L1 preadipocytes were induced to differentiate with IBMX, dexamethasone, and insulin in the absence or presence of indicated concentrations of caffeine. (A) Cell lysates were obtained after $48 \mathrm{~h}$ of induction and subjected to Western blot analyses using specific antibodies against each protein. Immunoblots are a representative image of three independent experiments. (B) mRNA expression was analyzed using RT-PCR. 


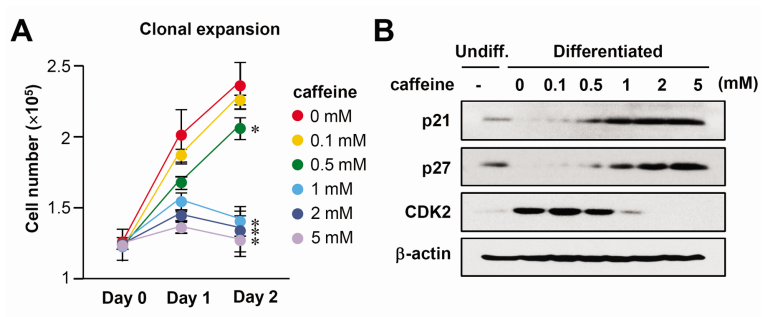

Fig. 3. The effect of caffeine on the mitotic clonal expansion process of adipocyte differentiation. Two-day post-confluent 3T3-L1 cells were incubated with the hormonal inducers in the absence or presence of various concentrations of caffeine. (A) Cell numbers were counted at the indicated time points after initiation of MDI-induced differentiation. Cells were trypsinized, aliquots were taken in triplicate, and then cells were counted using an ADAM cell counter. ${ }^{*} \mathrm{P}<0.01$ vs. $0 \mathrm{mM}$ caffeine group. (B) The protein levels of p21, p27, and cdk2 in these cells during differentiation (Day 2) were detected by immunoblot assay using specific antibodies. $\beta$-actin was used as a loading control.

the effect of caffeine on the proliferation of 3T3-L1 preadipocytes stimulated by the hormonal inducers of IBMX, dexamethasone, and insulin, designated as MDI. When 2 day post-confluent cells are incubated in a medium containing $\mathrm{MDI}$, the cell number increases over time for up to 2 days, because growth-arrested 3T3-L1 cells reenter the cell cycle and undergo approximately two rounds of cell division, called mitotic clonal expansion. The treatment of cells with 0.1 and 0.5 $\mathrm{mM}$ caffeine resulted in no change of mitotic clonal expansion but caffeine concentrations of 1,2 , and $5 \mathrm{mM}$ significantly abolished the increase in cell numbers at day 2 compared with MDI alone (Fig. 3A).

As previously reported (5), mitotic clonal expansion results in the detection of dividing cells (G2/M) after $24 \mathrm{~h}$ of induction. To examine how cell cycle events during mitotic clonal expansion are affected by caffeine, cells treated with various concentrations of caffeine were subjected to flow cytometry. While the cells with MDI synchronously entered S phase after $16 \mathrm{~h}$ of induction, cells with MDI plus caffeine clearly showed a decreased S phase population (Supplementary Fig. 1), indicating that caffeine blocks mitotic clonal expansion. We next examined whether caffeine altered the expression of cell cycle regulatory proteins. Caffeine treatment increased the expression of p21 and p27, the major regulators arresting cell cycle progression at the $\mathrm{G} 1 / \mathrm{S}$ checkpoint, whereas CDK2, the essential factor for G1/S transition, was downregulated (Fig. 3B). These results suggest that caffeine disturbed the mitotic clonal expansion at the level of $\mathrm{G} 1 / \mathrm{S}$ transition of the cell cycle.

In order to confirm the caffeine effect on adipogenesis, another preadipogenic cell line, 3T3-F442A cells, were tested. As shown in Supplementary Fig. 2, 3T3-F442A cells also showed similar results upon caffeine treatment, evident by oil-red-O staining, the expression of adipogenic markers, cell count, and FACS analysis.

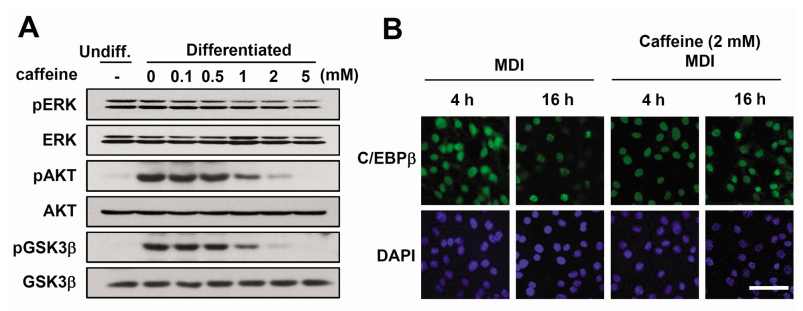

Fig. 4. The effect of caffeine on signaling molecules involved in mitotic regulation. Two-day post-confluent 3T3-L1 cells were incubated with the hormonal inducers in the presence or absence of various concentrations of caffeine for $48 \mathrm{~h}$. (A) Total cell lysates were prepared and analyzed by Western blot using the indicated phospho-specific antibodies. Western blot analysis of each total protein was done in parallel. Immunoblots are a representative image of three independent experiments. (B) Immunofluorescence analysis of C/EBP $\beta$. 3T3-L1 preadipocytes were induced to differentiate. After $4 \mathrm{~h}$ or $16 \mathrm{~h}$, cells were fixed and subjected to immunofluorescence analysis with antibody against C/EBP $\beta$ and 4',6-diamidino-2-phenylindole. Fluorescence images were obtained by confocal microscopy. Scale bar $=50 \mu \mathrm{m}$.

\section{Caffeine inhibits AKT/GSK3 $\beta$ signaling in differentiating 3T3-L1 preadipocytes}

Hormonal inducers, IBMX, dexamethasone, and insulin trigger the activation of several signaling pathways during adipogenesis (4). Among them, insulin or insulin-like growth factor (IGF)-I activates ERK and/or AKT signaling for the initial progression of adipogenesis, including mitotic clonal expansion $(23,24)$. Because caffeine inhibited the mitotic clonal expansion and adipocyte differentiation, we investigated whether caffeine affects these signaling pathways. When caffeine was added to differentiating 3T3-L1 cells, the phosphorylation of AKT and its downstream signal GSK3 $\beta$ (25) was reduced (Fig. $4 \mathrm{~A})$. However, ERK was not significantly affected by caffeine treatment (Fig. 4A). These results suggest that caffeine inhibits the AKT/GSK3 $\beta$-mediated signaling pathway stimulated by MDI.

While the expression of C/EBP $\beta$ remains unchanged (Fig. $2 \mathrm{~A})$, it is possible that the activation process of $\mathrm{C} / \mathrm{EBP} \beta$ might be affected by caffeine. C/EBP $\beta$ activation involves a serial event resulting in the characteristic "punctate" pattern at $16 \mathrm{~h}$ of differentiation in immunofluorescent staining (Fig. 4B), which was attenuated by the treatment of caffeine. This suggests that caffeine negatively affects the C/EBP $\beta$ activation process, as well as AKT signaling pathway.

\section{DISCUSSION}

The use of an anti-adipogenic agent derived from natural sources could be helpful in the prevention of obesity. Blocking of adipocyte differentiation is one of the anti-obesity strategies that fall under the category of modulating fat storage. In recent years, many groups have explored novel compounds derived from natural resources for their effect on adipogenic differentiation and gene regulation, often associated with obesity. 
For example, caffeine intake decreased diet-induced insulin resistance (20), inhibited lipid accumulation, and stimulated breakdown of accumulated triglyceride in adipocytes (21). Among them, a study by Nakayabashi et al. (21) concluded that $0.5 \mathrm{mM}$ caffeine had no obvious effect on adipocyte differentiation per se; however, in our study, we identified caffeine $(>1 \mathrm{mM})$ as an effective anti-adipogenic compound in cultured 3T3-L1 preadipocytes, preventing the adipocyte differentiation process by showing more comprehensive experiments; (1) oil-red-O staining, (2) differentiation markers including the negative regulators such as KLF2 and Pref-1, and (3) mitotic clonal expansion by cell count, FACS analysis, and the expression of $\mathrm{p} 21 / \mathrm{p} 27$.

The mechanism of action of anti-adipogenic agents should be carefully assessed, and importantly, studies should determine the stage of adipogenesis at which such agents are active. Adipogenesis requires a network of transcription factors and signaling pathways, which contribute to the serial gene expression for adipocyte differentiation and maturation. C/EBP $\beta$ is expressed immediately after exposure to adipogenic inducers, whereas PPAR $\gamma$ and C/EBP $\alpha$ expression are acquired 36-48 $\mathrm{h}$ after exposure. In this regard, it should be noted that $1 \mathrm{mM}$ caffeine did not affect C/EBP $\beta$ expression but repressed C/EBP $\alpha$ and PPAR $\gamma$ (Fig. 2A) expression. This suggests that caffeine works as an early adipogenic inhibitor, likely acting between C/EBP $\beta$ expression and C/EBP $/ \mathrm{PPAR} \gamma$ expression. $\mathrm{C} / \mathrm{EBP} \beta$ requires an activation process after its expression; first C/EBP $\beta$ is sequentially phosphorylated (11), resulting in dimerization of the phosphorylated $\mathrm{C} / \mathrm{EBP} \beta$, enabling it to bind its DNA binding element with the help of reactive oxygen species $(5,13)$. Therefore, in the presence of caffeine, C/EBP $\beta$ was expressed normally but might not be activated. This was proven by immunofluorescent study showing that caffeine attenuates the punctate pattern of $\mathrm{C} / \mathrm{EBP} \beta$ binding (Fig. 4B). Inhibitory factors of adipogenesis remained high with comparable treatment (Fig. 2B), also indicating that caffeine blocks an early adipogenic event. Consistently, caffeine inhibited the typical mitotic clonal expansion in our experiments (Fig. 3). We demonstrated that caffeine blocked the cell cycle at the G1/S transition and cell cycle protein analysis also supported G1 arrest by caffeine. Thus, our conclusion is that caffeine inhibits preadipocyte growth and differentiation at the initial stage, including C/EBP $\beta$ activation and mitotic clonal expansion of preadipocytes.

AKT mediates the signaling pathway of insulin or IGF-I in adipogensis, and in mature adipocytes, the activation of AKT is required for insulin-induced glucose transport as well as energy metabolism (24). Our results show that caffeine dose-dependently reduces the activation of AKT in 3T3-L1 adipocytes. It was reported that the involvement of AKT in the insulin or IGF-I signaling pathway is partly by phosphorylating GSK3 $\beta$ protein (26). Our data indicate that the treatment of caffeine decreases the phosphorylation of GSK3 $\beta$ in 3T3-L1 cells, suggesting that the AKT/GSK3 $\beta$ axis required for adipogenesis is disturbed by caffeine treatment. It is also possible that AKT-dependent inactivation of the Forkhead transcription factor (FKHR), which is essential for the progression of adipogenesis (27), is attenuated by caffeine. Taken together, our results revealed that caffeine-mediated suppression of adipogenesis is caused by attenuation of AKT and GSK3 $\beta$ phosphorylation resulting in decreased levels of C/EBP $\alpha$ and PPAR $\gamma$.

Caffeine-inhibited adipocyte differentiation was recently observed in mesenchymal stem cells (28), but the detailed molecular basis of this inhibition was not determined. Our results demonstrate that caffeine significantly suppresses the expression of adipogenic genes, thereby reducing lipid storage and accumulation in 3T3-L1 adipocytes. It should be noted that the concentration of caffeine used in this study is far beyond a physiologic range. The plasma concentration of caffeine was reported to be $0.54-1.94 \mathrm{mg} / \mathrm{L}$ in human consuming 0-3 cups of coffee a day (29). However, higher concentration of caffeine $(>1 \mathrm{mM})$ has been used in cell studies in vitro (30-32), as in the case of many other chemicals, hormones, and growth factors. For example, we use more than hundred folds of physiologic concentration of insulin for detecting glucose uptake, inducing differentiation, or observing an activation of signaling pathway. Thus, it is rather relevant to investigate the caffeine effect in vivo, fully considering a dose and duration of exposure together than to simply conclude it is only effective in high doses in cell culture system.

\section{MATERIALS AND METHODS}

For full details of all materials and methods, please refer to Supplementary Information

\section{ACKNOWLEDGEMENTS}

This work was supported by the National Research Foundation of Korea (NRF) Grants 2011-0030086, 2015R1A2A2A01004345, and 2013R1A1A1059527, funded by the Korea government, Ministry of Science, ICT and Future Planning (MSIP).

\section{REFERENCES}

1. Friedman JM (2004) Modern science versus the stigma of obesity. Nat Med 10, 563-569

2. Kopelman PG (2000) Obesity as a medical problem. Nature 404, 635-643

3. Spalding KL, Arner E, Westermark PO et al (2008) Dynamics of fat cell turnover in humans. Nature 453, 783-787

4. Cornelius P, MacDougald OA and Lane MD (1994) Regulation of adipocyte development. Annu Rev Nutr 14, 99-129

5. Lee H, Lee YJ, Choi H, Ko EH and Kim JW (2009) Reactive oxygen species facilitate adipocyte differentiation by accelerating mitotic clonal expansion. J Biol Chem 284, 10601-10609 
6. Otto TC and Lane MD (2005) Adipose development: from stem cell to adipocyte. Crit Rev Biochem Mol Biol 40, 229-242

7. Farmer SR (2006) Transcriptional control of adipocyte formation. Cell Metab 4, 263-273

8. Tontonoz P, Hu E and Spiegelman BM (1994) Stimulation of adipogenesis in fibroblasts by PPAR gamma 2, a lipid-activated transcription factor. Cell 79, 1147-1156

9. Cao Z, Umek RM and McKnight SL (1991) Regulated expression of three C/EBP isoforms during adipose conversion of 3T3-L1 cells. Genes Dev 5, 1538-1552

10. Lane MD, Tang QQ and Jiang MS (1999) Role of the CCAAT enhancer binding proteins (C/EBPs) in adipocyte differentiation. Biochem Biophys Res Commun 266, 677683

11. Tang QQ, Gronborg M, Huang $\mathrm{H}$ et al (2005) Sequential phosphorylation of CCAAT enhancer-binding protein beta by MAPK and glycogen synthase kinase 3 beta is required for adipogenesis. Proc Natl Acad Sci U S A 102, 97669771

12. Tang QQ and Lane MD (1999) Activation and centromeric localization of CCAAT/enhancer-binding proteins during the mitotic clonal expansion of adipocyte differentiation. Genes Dev 13, 2231-2241

13. Kim JW, Tang QQ, Li X and Lane MD (2007) Effect of phosphorylation and S-S bond-induced dimerization on DNA binding and transcriptional activation by C/EBPbeta. Proc Natl Acad Sci U S A 104, 1800-1804

14. Smas CM and Sul HS (1993) Pref-1, a Protein Containing Egf-Like Repeats, Inhibits Adipocyte Differentiation. Cell 73, 725-734

15. Banerjee SS, Feinberg MW, Watanabe M et al (2003) The Kruppel-like factor KLF2 inhibits peroxisome proliferatoractivated receptor-gamma expression and adipogenesis. J Biol Chem 278, 2581-2584

16. Lee $\mathrm{H}$, Kim HJ, Lee YJ, Lee MY, Choi $\mathrm{H}$ and Kim JW (2012) Kruppel-like factor KLF8 plays a critical role in adipocyte differentiation. PLoS One 7, e52474

17. Oishi Y, Manabe I, Tobe K et al (2005) Kruppel-like transcription factor KLF5 is a key regulator of adipocyte differentiation. Cell Metab 1, 27-39

18. Smith A (2002) Effects of caffeine on human behavior. Food Chem Toxicol 40, 1243-1255

19. Jacobson KA and Gao ZG (2006) Adenosine receptors as therapeutic targets. Nat Rev Drug Discov 5, 247-264

20. Conde SV, da Silva TN, Gonzalez C, Carmo MM, Monteiro EC and Guarino MP (2012) Chronic caffeine intake de- creases circulating catecholamines and prevents diet-induced insulin resistance and hypertension in rats. Brit J Nutr 107, 86-95

21. Nakabayashi $\mathrm{H}$, Hashimoto $\mathrm{T}$, Ashida $\mathrm{H}$, Nishiumi $\mathrm{S}$ and Kanazawa K (2008) Inhibitory effects of caffeine and its metabolites on intracellular lipid accumulation in murine 3T3-L1 adipocytes. Biofactors 34, 293-302

22. Akiba T, Yaguchi K, Tsutsumi K et al (2004) Inhibitory mechanism of caffeine on insulin-stimulated glucose uptake in adipose cells. Biochem Pharmacol 68, 1929-1937

23. Tang QQ, Otto TC and Lane MD (2003) Mitotic clonal expansion: a synchronous process required for adipogenesis. Proc Natl Acad Sci U S A 100, 44-49

24. Xu JF and Liao K (2004) Protein kinase B/AKT 1 plays a pivotal role in insulin-like growth factor-1 receptor signaling induced 3T3-L1 adipocyte differentiation. J Biol Chem 279, 35914-35922

25. Sutherland C, Leighton IA and Cohen P (1993) Inactivation of Glycogen-Synthase Kinase-3-Beta by Phosphorylation New Kinase Connections in Insulin and Growth-Factor Signaling. Biochem J 296, 15-19

26. Ross SE, Erickson RL, Hemati $\mathrm{N}$ and MacDougald OA (1999) Glycogen synthase kinase 3 is an insulin-regulated C/EBP alpha kinase. Mol Cell Biol 19, 8433-8441

27. Nakae J, Kitamura T, Kitamura Y, Biggs WH 3rd, Arden KC and Accili D (2003) The forkhead transcription factor Foxo1 regulates adipocyte differentiation. Dev Cell 4, 119-129

28. Su SH, Shyu HW, Yeh YT, Chen KM, Yeh H and Su SJ (2013) Caffeine inhibits adipogenic differentiation of primary adipose-derived stem cells and bone marrow stromal cells. Toxicol in Vitro 27, 1830-1837

29. de Leon J, Diaz FJ, Rogers T et al (2003) A pilot study of plasma caffeine concentration in a US sample of smoker and nonsmoker volunteers. Prog Neuropshychopharmacol Biol Psychiatry 27, 165-171

30. Hashimoto T, He Z, Ma WY et al (2004) Caffeine inhibits cell proliferation by G0/G1 phase arrest in JB6 cells. Cancer Res 64, 3344-3349

31. Okano J, Nagahara T, Matsumoto $K$ and Murawaki $Y$ (2008) Caffeine inhibits the proliferation of liver cancer cells and activates the MEK/ERK/EGFR signalling pathway. Basic Clin Pharmacol Toxicol 102, 543-551

32. Sinchai T, Plasen S, Sanvarinda Y et al (2011) Caffeine potentiates methamphetamineinduced toxicity both in vitro and in vivo. Neurosci Lett 502, 65-69 